\title{
1 Genomic signature of accelerated evolution in a saline-alkaline lake-dwelling
}

\section{Schizothoracine fish}

4 Chao Tong ${ }^{1, *}$, Miao $\mathrm{Li}^{2}$

$5 \quad{ }^{1}$ Department of Biology, University of Pennsylvania, Philadelphia, PA, USA

$6 \quad{ }^{2}$ Center for Advanced Retinal and Ocular Therapeutics, Perelman School of Medicine, University of

7 Pennsylvania, Philadelphia, PA, USA

8

$9 *$ Corresponding authors: Chao Tong

10 Phone: +1-215-452-8712

11 Email: tongchao1990@gmail.com

12 ORCID: https://orcid.org/0000-0001-5202-5507

13 Miao Li: $\underline{\text { limiao1199@gmail.com }}$

14 


\section{Abstract}

16 Tibetan Plateau imposes extremely inhospitable environment on most wildlife. Besides the harsh aquatic

17 environment including hypoxia and chronic cold, high salinity and alkalinity is an increasing threat to

18 Tibetan endemic fishes. Previous genome-wide studies identified key genes contributed to highland fish

19 adaptation to hypoxia and long-term cold, while our understanding of saline and alkaline adaptation in

20 Tibetan fish remains limited. In this study, we performed a comparative genomics analysis in a saline

21 lake-dwelling highland fish Gymnocypris przewalskii, aimed to identify candidate genes that contributed

22 to saline and alkaline adaptation. We found elevated genome-wide rate of molecular evolution in $G$.

23 przewalskii relative to lowland teleost fish species. In addition, we found nine genes encoding biological

24 macromolecules associated with ion transport functions underwent accelerated evolution in $G$.

25 przewalskii, which broadly expressed across kidney, gill, liver, spleen, brain and muscle tissues.

26 Moreover, we found putative evidence of ion transport under selection were interacted by co-expression

27 in G. przewalskii adaptation to high salinity and alkalinity environment of Lake Qinghai. Taken together,

28 our comparative genomics study identified a set of rapidly evolving ion transport genes and

29 transcriptomic signatures in Schizothoracine fish adaptation to saline and alkaline environment on the

30 Tibetan Plateau.

32 Keywords: Comparative genomics, Schizothoracinae, Accelerated evolution, Saline and alkaline

33 adaptation 


\section{Introduction}

36 Environments may shape the genetic landscape of wildlife that inhabit them [1]. The world's largest and

37 highest highland Tibetan Plateau had undergone continuous uplift during the India-Asia collision since

38 about 45 million years ago, which triggered numerous environmental changes [2,3]. As elevation above

39 sea level increases, a decrease in barometric pressure results in fewer oxygen molecules in the air, which

40 causes hypoxia. Besides, other challenging environments high-altitude dwelling wildlife have

41 encountered are the long-term low temperature and high ultraviolet radiation [4,5]. Understanding how

42 organism adapt to their dwelling environment is central to answering many ecological and evolutionary

43 questions, but it remains a formidable task to fully uncover the mechanisms of adaptive process [6].

44 Adaptation at the molecular level can occur by adaptive mutation in key genes over prolonged

45 evolutionary time scales [7]. Recent genome-wide studies have identified key genes associated with

46 hypoxia response and energy metabolism in Tibetan terrestrial animals adaptation to high altitude [8-10].

47 Nevertheless, the adaptive mechanism of Tibetan aquatic organisms to highland water environment is yet

48 well-studied [11].

50 Unlike Tibetan terrestrial animal, the draft genomes of very few Tibetan aquatic organisms had been

51 sequenced $[12,13]$, the genomic basis of aquatic animals adaptation to water environments at high altitude

52 still remain largely unknown. The Schizothoracine fishes are the predominant aquatic fauna on the

53 Tibetan Plateau, which had evolved specific phenotypic characteristics to adapt to the harsh aquatic

54 environments, such as hypoxia, chronic cold and high salinity and alkalinity. Comparative genomics

55 approaches have the power to facilitate investigation of the genomic basis of evolution and adaptation

56 [14]. Recent comparative genomics studies based on transcriptomic data of several Schizothoracine

57 species have identified a number of candidate genes that underwent positive selection during the long-

58 term adaptive processes to harsh environments on the Tibetan Plateau, such as BYSL and HSF1

59 associated with hypoxia response [15] and ND1, ATAD2 and ARL3 that involved into cold response

$60[16,17]$. Notably, an increasing number of lakes are existing or towards saline and alkaline due to the

61 geological evolution and global climate changes on the Tibetan Plateau [3,18]. For instance, Lake

62 Qinghai, the largest salt lake in China, is highly saline (up to 13\%o) and alkaline (up to pH 9.4) water

63 environment. It is also a typical salt lake with unusually high sodium, potassium and magnesium

64 concentration [18,19]. Intriguingly, Lake Qinghai used to be freshwater and connected to the Yellow

65 River, while was late separated with the upper reaches of the Yellow River during the geological event

66 "Gonghe Movement" (approximately 15 mya) [19,20]. Moreover, the increasing of water salinization is a 
67 growing threat to freshwater fish species [21,22]. Tibetan freshwater endemic fishes are long suffering

68 these harsh conditions challenges [11,18]. The main focus of the genetic mechanism of highland

69 adaptation in Tibetan fish are still on hypoxia and chronic cold response [15,23-25]. However, the

70 genomic signature of high salinity and alkalinity adaptation in Schizothoracine fish have yet to be

71 comprehensively determined.

73 Unlike other broadly distributed Schizothoracinae fish species, Gymnocypris przewalskii is only endemic

74 to Lake Qinghai [19,20,26]. Past studies suggested that G. przewalskii has gradually evolved from

75 freshwater fish to tolerate high salinity and alkalinity of Lake Qinghai during the early to late Holocene

76 [26]. Because of the unique evolutionary history in Lake Qinghai at high altitude, G. przewalskii provides

77 an exceptional model to investigate the genetic mechanisms underlying adaptation to high salinity and

78 alkalinity environment on the Tibetan Plateau. In this study, we performed a comparative genomics

79 analysis and identified a set of ion transport genes that showing strong signals of rapidly evolving in $G$.

80 przewalskii. Specifically, we used the de novo transcriptome assemblies from multiple tissue RNA-seq

81 data and five well-annotated teleost fish genomes for comparison. In addition, we estimated the genome-

82 wide nucleotide substitution rate of each fish species. Moreover, using the tissue-transcriptomics, we

83 characterized the expression patterns of rapidly evolving ion transport genes in kidney, gill, liver, spleen,

84 brain and muscle of highland fish, G. przewalskii.

\subsection{Data collection and transcriptome assembly}

88 We downloaded the transcriptome sequencing data of Schizothoracine fish G. przewalskii from NCBI

89 SRA database (https://www.ncbi.nlm.nih.gov/sra). Specifically, we collected six tissues transcriptomics

90 including kidney, gill, liver, spleen, brain and muscle of G. przewalskii (supplementary table S1). At first,

91 we checked the quality of the raw sequencing reads using FastQC v 0.11.8

92 (https://www.bioinformatics.babraham.ac.uk/projects/fastqc/). Sequencing adapters and reads with a

93 quality score $<20$ were trimmed with Trimmomatic v.0.36 [27], resulting in clean sequencing reads.

94 Then, we performed de novo transcriptome assembly using Trinity v2.8.5 [28] with default parameters.

95 After assembly, the redundant transcripts were removed using CD-HIT v4.8.1 [29] with the threshold of

960.90 , and only the longest transcript under each cluster was extracted as unigene (unique gene). Next, we

97 predicted the open reading frame (ORF) of each unigene using TransDecoder 
98 (https://github.com/TransDecoder/TransDecoder). Finally, we translated the nucleotide sequences of

99 protein-coding genes from the assemblies of G. przewalskii into amino acid sequences using an in-house-

100 developed perl script.

\subsection{Orthologs identification and sequence alignment}

103 We included five well-annotated teleost fish genomes for comparative genomics analysis and downloaded

104 from Ensembl database (http://useast.ensembl.org/index.html), including zebrafish (Danio rerio), tilapia

105 (Oreochromis niloticus), medaka (Oryzias latipes), fugu (Takifugu rubripes) and cod (Gadus morhua).

106 Then, we built a local protein database including the sequences from above five fish genomes and $G$.

107 przewalskii transcriptome assemblies. Next, we downloaded the curated orthology map of Actinopterygii

108 (ray-finned fish) from OrthoDB database (release 8) [30] which contains 21,952 orthologous gene groups

109 information. Of these seed orthologous groups in HaMStR v13.2.6 [31], we identified the orthologs in

110 each fish species with E-values of less than $10^{-20}$. Moreover, we aligned and trimmed the protein

111 sequences of the orthologous groups using PRANK [32] and MATFF v 7.450 [33], and trimmed using

112 trimAl [34] with the parameter "-automated1". Among the identified orthologs, we identified one-to-one,

113 one-to-many and many-to-many orthologs in each fish species. For each 1:1 orthologous pair (i.e. genes

114 for which only one gene from each species matches the given OrthoDB orthologous gene group), we only

115 selected the longest transcript associated with the gene for each pair of species as putative single-copy

116 ortholog. Finally, we identified the core single-copy orthologs that were shared by above six fish species.

\subsection{Genome-scale concatenation and coalescent based dataset construction}

119 We performed the alignment of core shared single-copy orthologs of six fish species using MUSCLE [35] with default parameters and trimmed using trimAl v1.2 [34] with parameter "-automated1". In addition, we filtered the core shared single-copy orthologs with strict constraints, including length (minimum 200 aa) and sequence alignment (maximum missing data 50\% in alignments). Next, we prepared two types of datasets after filtration. Firstly, we concatenated all core shared single-copy genes from each species into one-line sequence as a supergene using a custom python script (genome-scale concatenation-based dataset), respectively. Secondly, we conducted a genome-scale coalescent-based dataset including core shared single-copy genes from each species. 
We used the clipped species tree (Figure 1A) including above six fish species from a previous study [16]. To estimate lineage-specific evolutionary rates for each fish species, we aligned core shared single-copy orthologs using MUSCLE [35], derived nucleotide alignments from protein alignments using PAL2NAL v14 [36], and estimated pairwise non-synonymous to synonymous substitutions (dN/dS) of nucleotide alignments using the CodeML package in PAML 4.7a [37]. Specifically, we used the free-ratio model ("several $\omega$ ratio") to calculate the ratio of $\mathrm{dN}$ to $\mathrm{dS}$ nucleotide changes separately for each ortholog and a concatenation of all alignments of single-copy orthologs from above six fish species. Parameters, including $\mathrm{dN}, \mathrm{dS}, \mathrm{dN} / \mathrm{dS}, \mathrm{N}^{*} \mathrm{dN}$, and $\mathrm{S} * \mathrm{dS}$ values, were estimated for each branch, and genes were discarded if $\mathrm{N} * \mathrm{dN}$ or $\mathrm{S} * \mathrm{~d} \mathrm{~S}<1$, or $\mathrm{dS}>2$, following previous studies $[11,16,17]$.

We sought to identify a set of genes with elevated $\mathrm{dN} / \mathrm{dS}$ in G. przewalskii relative to other five fish species. At first, we ran two branch models using CodeML package in PAML 4.7a [37] to identify rapidly evolving genes (REGs) in G. przewalskii lineage with corresponding nucleotide alignments, specifically with the null model assuming that all branches have been evolving at the same rate and the alternative model allowing the focal foreground branch (G. przewalskii) to evolve under a different evolutionary rate.

144 Next, we used a likelihood ratio test (LRT) in R software, package MASS with $\operatorname{df} \square=\square 1$ to discriminate 145 between the alternative model and the null model for each single-copy orthologs in the genesets. We only 146 considered the genes as rapidly evolving with a significantly faster rate in the foreground branch if the

147 adjusted $P$ value $\square<\square 0.05$ and higher $\mathrm{dN} / \mathrm{dS}$ in the focal foreground branch than focal background 148 branches (other four fish species). Finally, we annotated the rapidly evolving genes with gene ontology 149 (GO) function category using R software, package topGO [38].

\subsection{Gene expression analysis}

152 After preparation of clean reads from six tissue-transcriptomics (kidney, gill, liver, spleen, brain and 153 muscle) of G. przewalskii, we performed the transcript quantification by mapping all clean reads to the 154 transcriptome assemblies using RSEM v1.3.1 [39] to obtain expected counts and fragments per kilobase 155 million (FPKM). In addition, we primarily focused on the expression pattern of rapidly evolving genes 156 (REGs), and calculated the FPKM value of each REG in each tissue. At last, we annotated the 157 differentially expressed REGs by gene ontology using R software, package TopGO [38]. 


\subsection{Transcriptome assemblies and orthologs}

161 By pooling six tissue-transcriptome sequencing data, the de novo transcriptome assembly of $G$.

162 przewalskii yielded 409,685 transcripts, with an N50 of 1,796 bp and an average length of $986 \mathrm{bp}$. After

163 removing redundant isoforms and extraction of longest isoform among alternative transcripts, a total of

164357,601 unigenes were obtained, with an N50 of 3,079 bp and a mean length of 1,992 bp. After protein-

165 coding gene prediction with TransDecoder, we totally obtained 137,539 unigenes with full or partial

166 length of gene coding regions (CDS) in G. przewalskii.

After identification of orthologs according to the curated orthologous gene groups of Actinopterygii in each fish species, we obtained a total of 74,107 putative orthologs in 16,379 orthologous gene groups (Table 1). After strict 1:1 ortholog selection, we identificated 16,379 longest orthologs that represent their gene groups as unique ortholog (Table 1). In addition, we eventually obtained core 10,260 orthologs that shared by all six fish species, making them suitable for comparative genomics analysis.

\subsection{Genome-wide nucleotide substitution rate}

175 After estimation of the nucleotide substitution rates of each branch that represented each fish species 176 based on 6,742 core shared single-copy orthologs, we found that Schizothoracine fish G. przewalskii had 177 elevated terminal genome-wide concatenation-based dN/dS compared to other five fish species (Figure 178 1B). Furthermore, we also found similar elevated pattern of genome-wide coalescent-based dN/dS in $G$. 179 przewalskii relative to other species (Figure 1C).

182 A set of genes with the signature of an increase rate of non-synonymous changes and underwent 183 accelerated evolution, namely rapidly evolving genes. We identified 466 putative rapidly evolving single184 copy orthologs (REGs) in G. przewalskii (supplementary table S2). Among this set of genes, the most 185 interesting finding was REGs included genes associated with ion transport functions. This group included 186 sodium channel subunit beta-3 (SCN3B), solute carrier family 13 member 3 (SLC13A3), transmembrane 187 protein $175\left(\mathrm{TMEM}^{275)}\right.$ ) and $\mathrm{H}\left({ }^{+}\right) / \mathrm{Cl}\left({ }^{-}\right)$exchange transporter 7 (CLCN7) (Table 2). Moreover, we found 188 a number of REGs associated with mitochondrial function and also involved ion transport process, such as sodium/potassium-transporting ATPase subunit beta-2 (ATP1B2), calcium uniporter protein (MCU) 
and calcium uptake protein 2 (MICU2) (Table 2). Besides the ion transport genes, we found a large number of genes involved energy metabolism function, such as ATP5c1 and ATP5b associated with ATP binding and oxidative phosphorylation process (supplementary table S2). Although previous comparative genomics studies with highland fish identified several candidate genes with the signals of positive selection $[15,23,24]$, here, we failed to identify any gene that potentially associated with hypoxia response.

\subsection{Tissue expression patterns of rapidly evolving ion transport genes}

After mapping the clean reads from six tissue-transcriptome sequencing data, we obtained the expression level of each unigenes based on FPKM value (supplementary table S3). We focused on the expression pattern of ion transport genes with the signature of accelerated evolution. Notably, we found eight rapidly evolving ion transport genes were broadly expressed in six tissues, except transient receptor potential cation channel subfamily V member 6 (TRPV6) that only expressed in liver (Figure 2A). In addition, the hierarchical clustering which illustrated by heatmap indicated that four genes (ATP1B2, MCU, CLCN7 and NALCN) and another five genes (MICU2, SCN3B, TMEM175, TRPV6 and SLC13A3) showed similar tissues expression patterns, respectively (Figure 2B). Moreover, gene ontology (GO) enrichment analysis showed that this set of differentially expressed REGs was significantly enriched multiple functions, such as ion transport (GO:0006811, $P=0.00031$ ), sodium ion transport $(\mathrm{GO}: 0006814, P=0.00047)$, calcium ion transport (GO:0006816, $P=0.00056$ ), chloride transport (GO:0006821, $P=0.00067$ ), response to $\mathrm{pH}$ (GO:0009268, $P=0.00069)$ and response to calcium ion (GO:0051592, $P=0.00078$ ) (Figure 2C).

\section{4. Discussion}

212 Over the past few years, transcriptome-based assembly approach enables comparative genomics studies 213 widely employed in many Tibetan endemic organisms to provide insights of highland adaptation [15-

$21417,23,40,41]$. Unlike whole genome data, although transcriptome sequencing is an effective and

215 accessible approach to initiate comparative genomic analyses on non-model organisms [28], it still can

216 not cover the whole protein coding gene repertoire of one species. Previous transcriptome studies on

217 Tibetan fishes mainly included one or two tissues [17,40,41], our present study included six tissues

218 (kidney, gill, liver, spleen, brain and muscle) RNA-seq data of G. przewalskii and generated much more

219 transcripts than previously assemblies [16,40]. In addition, we used curated orthology mapping approach

220 [42] and identified more than 15,000 pairwise orthologous genes in each fish species and over 10,000 
core single-copy orthologs shared by six species, which included much more orthologs than our early studies $[16,17]$. These putative single-copy orthologs are the important bases for comparative genomic analysis. Notably, most high-altitude dwelling Schizothoracine fish species are polyploidy with high complexity and large size of genomes, the whole genome data is long lacking [11]. Therefore, comparative genomic analysis based on transcriptome assemblies of Schizothoracine fish will still be the tendency in recent years.

Our present study pinpointed that highland fish, G. przewalskii has elevated rate of molecular evolution $(\mathrm{dN} / \mathrm{dS})$ on both concatenation and coalescent genomic-scales compared with lowland fish species, indicating that G. przewalskii may be under rapidly evolving. Not surprisingly, this result was consistent with previous studies in other Tibetan endemic fish species [15-17,23,41]. In addition, this finding highlighted animals endemic to the Tibetan Plateau underwent accelerated evolution (high $\mathrm{dN} / \mathrm{dS}$ ) relative to low-altitude dwelling organisms $[9,10]$. Furthermore, species inhabit similar ecological niches may be shaped by convergent evolution to form physiological or morphological similarities [43]. Like other Tibetan terrestrial wildlife, our finding implied that the elevation of genome-wide nucleotide substitution rate is one of adaptive process of G. przewalskii to harsh environment in Lake Qinghai, including the increasing of water salinization.

Accelerated evolution at molecular level may be reflected by an increased rate of non-synonymous changes within genes involved in adaptation [44]. Our present comparative study identifies a set of rapidly evolving genes associated with ion transport function in G. przewalskii. These genes encoded biological macromolecules which mainly functioning in sodium ion transport, calcuim ion transport, chloride transport and response to $\mathrm{pH}$ processes. This result is consistent with findings in an extremely

244 alkaline environment dwelling fish, Leuciscus waleckii [45], indicating that the alkaline environment of 245 both Lake Qinghai and Lake Dali Nur spurred accelerated evolution of ion transport genes in both fish 246 species. Notably, the rapidly evolving gene repertoire of G. przewalskii included SLC13A3, TMEM175 and CLCN7. Solute carrier (SLC) is a family that encoded transmembrane transporters for inorganic ions,

248 amino acids, neurotransmitters, sugars, purines and fatty acids, and other solute substrates [46]. Past

249 evidence suggested that the adaptive evolution of solute carrier genes contribute to high salinity and 250 alkalinity adaptation in fishes [45-47]. Specifically, SLC13 is a subfamily of sodium sulphate/carboxylate 251 cotransporters [48]. Moreover, CLC gene is a family of $\mathrm{H}^{+} / \mathrm{Cl}^{-}$exchange transporter that mediate 252 transmembrane $\mathrm{Cl}-$ transport [49]. In addition, previous study suggested that TMEM175 is involved in 
potassium channel activity [50]. Therefore, we suggested that ion transport genes underwent rapidly evolving is another adaptation strategy for G. przewalskii to cope with the severe saline and alkaline stress in Lake Qinghai.

Previous studies identified a number of genes under accelerated evolution that mainly involved energy metabolism pathways [11,15-17,23-25]. Compared with a few rapidly evolving ion transport genes that were found in G. przewalskii, this present study identified a number of candidate genes that related to energy metabolism and contributed to long-term cold adaptation. Gene associated with energy metabolism showing signs of adaptive evolution is one of the genomic signatures that had been identified in Tibetan Plateau dwelling animals [16,17]. Our finding is consistent with previous comparative genomics studies in highland fishes as well [15-17]. A set of genes functioning in energy supply and metabolism were under accelerated evolution in G. przewalskii, such as ATP5b and ATP5c, ATP synthase subunit beta. In addition, although hypoxia adaptation is one of the significant adaptive processes contributed to highland adaptation in endemic animals that dwelt at high altitude [10,16,17], we still were not able to identify any rapidly evolving genes associated with hypoxia response function in present study. Indeed, there is a long controversial issue about hypoxic environment and hypoxia response for Tibetan fish species. Obviously, more physiological, ecological and genomic analysis were required to reveal the mechanism of highland fish adaptation to hypoxia.

A set of previous studies indicated that fish gills, kidney, liver and spleen are key tissues that contributed to saline and alkaline tolerance [51,52]. Using tissue-transcriptomic data, we characterized the expression profiles of six tissue types. Most of rapidly evolving ion transport genes have broad expression patterns across all tissues. In addition, these broadly expressed ion transport genes were mainly associated with sodium ion transport, chloride transport and response to $\mathrm{pH}$ function by gene ontology annotation. This finding indicates that ion transport genes in G. przewalskii experiencing accelerated evolution may have general functions and involve into multiple biological processes. Furthermore, we found a set of rapidly evolving ion transport genes that involved distinct pathways showed the similar tissue expression patterns. That is said, these ion transport genes under selection were putatively interacted by cooperation in G. przewalskii adaptation to high salinity and alkalinity environment of Lake Qinghai. Therefore, this

282 finding indicated that future Schizothoracine fish comparative genomics study, including increasing 283 sequencing and function assay, can further clarity the molecular basis of saline and alkaline adaptation of 284 high-altitude dwelling fishes. 


\section{Conclusion}

287 We used comparative genomics based on the de novo assemblies from pooled six tissues transcriptomes

288 to identify the genomic signature of saline and alkaline adaptation in a highland fish, G. przewalskii.

289 These putative genomic signatures included: (1) Schizothoracine fish, G. przewalskii had an elevated

290 genome-wide nucleotide substitution rate than lowland teleost fish species; (2) a number of genes

291 associated with ion transport and energy metabolism functions were found in G. przewalskii with elevated

292 molecular evolutionary rate (dN/dS) showing the signature of rapidly evolving; (3) most of rapidly

293 evolving ion transport genes associated with sodium ion transport, calcium ion transport and chloride

294 transport were broadly expressed in kidney, gill, liver, spleen, brain and muscle of G. przewalskii; (4) A

295 set of rapidly evolving ion transport genes exhibited similar tissue expression patterns and were interacted

296 by co-expression in G. przewalskii. Altogether, our present study will provide the genomic signatures of

297 rapidly evolving ion transport genes, and gain the insights into the saline and alkaline adaptation of high-

298 altitude dwelling fishes.

\section{Acknowledgments}

301 This work was funded by the Field Research Fund of University of Pennsylvania Biology Department.

\section{References}

304 [1] K.E. Witt, E. Huerta-Sánchez, Convergent evolution in human and domesticate adaptation to highaltitude environments, Philos. Trans. R. Soc. Lond. B Biol. Sci. 374 (2019) 20180235.

[2] J. Li, X. Fang, Uplift of the Tibetan Plateau and environmental changes, Chinese Science Bulletin. 44 (1999) 2117-2124.

[3] A. Favre, M. Päckert, S.U. Pauls, S.C. Jähnig, D. Uhl, I. Michalak, A.N. Muellner-Riehl, The role of the uplift of the Qinghai-Tibetan Plateau for the evolution of Tibetan biotas, Biol. Rev. Camb. Philos. Soc. 90 (2015) 236-253.

311 [4] L.B. Scheinfeldt, S.A. Tishkoff, Living the high life: high-altitude adaptation, Genome Biol. 11 (2010) 133. 
[5] Z. An, K. John, P. Warren, P. Stephen, Evolution of Asian monsoons and phased uplift of the Himalaya-Tibetan plateau since Late Miocene times, Nature. 411 (2001) 62-66.

[6] N.G.C. Smith, A. Eyre-Walker, Adaptive protein evolution in Drosophila, Nature. 415 (2002) 10221024.

[7] H.A. Orr, The genetic theory of adaptation: a brief history, Nat. Rev. Genet. 6 (2005) 119-127.

[8] J.-T. Li, Y.-D. Gao, L. Xie, C. Deng, P. Shi, M.-L. Guan, S. Huang, J.-L. Ren, D.-D. Wu, L. Ding, Z.-Y. Huang, H. Nie, D.P. Humphreys, D.M. Hillis, W.-Z. Wang, Y.-P. Zhang, Comparative genomic investigation of high-elevation adaptation in ectothermic snakes, Proc. Natl. Acad. Sci. U.

[10] Q. Qiu, G. Zhang, T. Ma, W. Qian, J. Wang, Z. Ye, C. Cao, Q. Hu, J. Kim, D.M. Larkin, L. Auvil,

[9] Y. Qu, H. Zhao, N. Han, G. Zhou, G. Song, B. Gao, S. Tian, J. Zhang, R. Zhang, X. Meng, Y. Zhang, Y. Zhang, X. Zhu, W. Wang, D. Lambert, P.G.P. Ericson, S. Subramanian, C. Yeung, H. Zhu, Z. Jiang, R. Li, F. Lei, Ground tit genome reveals avian adaptation to living at high altitudes in the Tibetan plateau, Nat. Commun. 4 (2013) 2071. B. Capitanu, J. Ma, H.A. Lewin, X. Qian, Y. Lang, R. Zhou, L. Wang, K. Wang, J. Xia, S. Liao, S. Pan, X. Lu, H. Hou, Y. Wang, X. Zang, Y. Yin, H. Ma, J. Zhang, Z. Wang, Y. Zhang, D. Zhang, T. Yonezawa, M. Hasegawa, Y. Zhong, W. Liu, Y. Zhang, Z. Huang, S. Zhang, R. Long, H. Yang, J. Wang, J.A. Lenstra, D.N. Cooper, Y. Wu, J. Wang, P. Shi, J. Wang, J. Liu, The yak genome and adaptation to life at high altitude, Nat. Genet. 44 (2012) 946-949. chromosome $\square$ level genome assembly of Triplophysa tibetana, a fish adapted to the harsh high $\square$ altitude environment of the Tibetan plateau, Molecular Ecology Resources. 19 (2019) 10271036. 
[14] J. Stapley, J. Reger, P.G.D. Feulner, C. Smadja, J. Galindo, R. Ekblom, C. Bennison, A.D. Ball, A.P. Beckerman, J. Slate, Adaptation genomics: the next generation, Trends Ecol. Evol. 25 (2010) 705-

345 712.

[15] L. Yang, Y. Wang, Z. Zhang, S. He, Comprehensive transcriptome analysis reveals accelerated genic evolution in a Tibet fish, Gymnodiptychus pachycheilus, Genome Biol. Evol. 7 (2014) 251-261.

[16] C. Tong, T. Fei, C. Zhang, K. Zhao, Comprehensive transcriptomic analysis of Tibetan Schizothoracinae fish Gymnocypris przewalskii reveals how it adapts to a high altitude aquatic life, BMC Evolutionary Biology. 17 (2017) 74.

[17] C. Tong, F. Tian, K. Zhao, Genomic signature of highland adaptation in fish: a case study in Tibetan Schizothoracinae species, BMC Genomics. 18 (2017) 948.

[18] M. Zheng, An Introduction to Saline Lakes on the Qinghai-Tibet Plateau, Springer Science \& Business Media, 1997.

[19] S. Zhu, Y. Wu, Study of fish fauna in Qinghai Lake, Fish Fauna and Biology of Naked Carp in Qinghai Lake. (1975) 9-26.

[20] Y. Wu, The Fishes of the Qinghai-Tibetan Plateau, Sichuan Publishing House of Science \& Technology, 1992.

[21] E.R. Herbert, P. Boon, A.J. Burgin, S.C. Neubauer, R.B. Franklin, M. Ardón, K.N. Hopfensperger, L.P.M. Lamers, P. Gell, A global perspective on wetland salinization: ecological consequences of a growing threat to freshwater wetlands, Ecosphere. 6 (2015) art206.

[22] W.D. Hintz, R.A. Relyea, A salty landscape of fear: responses of fish and zooplankton to freshwater salinization and predatory stress, Oecologia. 185 (2017) 147-156.

[23] Y. Wang, L. Yang, K. Zhou, Y. Zhang, Z. Song, S. He, Evidence for Adaptation to the Tibetan Plateau Inferred from Tibetan Loach Transcriptomes, Genome Biol. Evol. 7 (2015) 2970-2982.

[24] L. Guan, W. Chi, W. Xiao, L. Chen, S. He, Analysis of hypoxia-inducible factor alpha polyploidization reveals adaptation to Tibetan Plateau in the evolution of schizothoracine fish, BMC Evol. Biol. 14 (2014) 192.

[25] Q. Xu, C. Zhang, D. Zhang, H. Jiang, S. Peng, Y. Liu, K. Zhao, C. Wang, L. Chen, Analysis of the erythropoietin of a Tibetan Plateau schizothoracine fish (Gymnocypris dobula) reveals enhanced 
cytoprotection function in hypoxic environments, BMC Evol. Biol. 16 (2016) 11.

371

372

373

374

375

376

377

378

379

380

381

382

383

384

385

[26] K. Zhao, Z. Duan, G. Yang, Z. Peng, S. He, Y. Chen, Origin of Gymnocypris przewalskii and phylogenetic history of Gymnocypris eckloni (Teleostei: Cyprinidae), Prog. Nat. Sci. 17 (2007) 520528.

[27] A.M. Bolger, M. Lohse, B. Usadel, Trimmomatic: a flexible trimmer for Illumina sequence data, Bioinformatics. 30 (2014) 2114-2120.

[28] M.G. Grabherr, B.J. Haas, M. Yassour, J.Z. Levin, D.A. Thompson, I. Amit, X. Adiconis, L. Fan, R. Raychowdhury, Q. Zeng, Z. Chen, E. Mauceli, N. Hacohen, A. Gnirke, N. Rhind, F. di Palma, B.W. Birren, C. Nusbaum, K. Lindblad-Toh, N. Friedman, A. Regev, Full-length transcriptome assembly from RNA-Seq data without a reference genome, Nat. Biotechnol. 29 (2011) 644-652.

[29] L. Fu, B. Niu, Z. Zhu, S. Wu, W. Li, CD-HIT: accelerated for clustering the next-generation sequencing data, Bioinformatics. 28 (2012) 3150-3152.

[30] E.V. Kriventseva, F. Tegenfeldt, T.J. Petty, R.M. Waterhouse, F.A. Simão, I.A. Pozdnyakov, P. Ioannidis, E.M. Zdobnov, OrthoDB v8: update of the hierarchical catalog of orthologs and the underlying free software, Nucleic Acids Res. 43 (2015) D250-6.

[31] I. Ebersberger, S. Strauss, A. von Haeseler, HaMStR: profile hidden markov model based search for orthologs in ESTs, BMC Evol. Biol. 9 (2009) 157.

[32] A. Löytynoja, N. Goldman, An algorithm for progressive multiple alignment of sequences with insertions, Proc. Natl. Acad. Sci. U. S. A. 102 (2005) 10557-10562.

[33] K. Katoh, D.M. Standley, MAFFT multiple sequence alignment software version 7: improvements in performance and usability, Mol. Biol. Evol. 30 (2013) 772-780.

[34] S. Capella-Gutiérrez, J.M. Silla-Martínez, T. Gabaldón, trimAl: a tool for automated alignment trimming in large-scale phylogenetic analyses, Bioinformatics. 25 (2009) 1972-1973.

[35] R.C. Edgar, MUSCLE: a multiple sequence alignment method with reduced time and space complexity, BMC Bioinformatics. 5 (2004) 113.

[36] M. Suyama, D. Torrents, P. Bork, PAL2NAL: robust conversion of protein sequence alignments into the corresponding codon alignments, Nucleic Acids Res. 34 (2006) W609-12. 
[37] Z. Yang, PAML 4: phylogenetic analysis by maximum likelihood, Mol. Biol. Evol. 24 (2007) 15861591.

[38] A. Alexa, J. Rahnenfuhrer, topGO: enrichment analysis for gene ontology, R Package Version. 2 (2010) 2010.

[39] B. Li, C.N. Dewey, RSEM: accurate transcript quantification from RNA-Seq data with or without a reference genome, BMC Bioinformatics. 12 (2011) 323.

[40] R. Zhang, A. Ludwig, C. Zhang, C. Tong, G. Li, Y. Tang, Z. Peng, K. Zhao, Local adaptation of Gymnocypris przewalskii (Cyprinidae) on the Tibetan Plateau, Scientific Reports. 5 (2015) 9780.

[41] X. Ma, W. Dai, J. Kang, L. Yang, S. He, Comprehensive Transcriptome Analysis of Six Catfish Species from an Altitude Gradient Reveals Adaptive Evolution in Tibetan Fishes, G3 . 6 (2015) 141148.

[42] C. Tong, G.M. Najm, N. Pinter-Wollman, J.N. Pruitt, T.A. Linksvayer, Comparative genomics identifies putative signatures of sociality in spiders, bioRxiv. (2019) 797241. doi:10.1101/797241.

[44] G. Zhang, C. Li, Q. Li, B. Li, D.M. Larkin, C. Lee, J.F. Storz, A. Antunes, M.J. Greenwold, R.W. Meredith, A. Ödeen, J. Cui, Q. Zhou, L. Xu, H. Pan, Z. Wang, L. Jin, P. Zhang, H. Hu, W. Yang, J. Hu, J. Xiao, Z. Yang, Y. Liu, Q. Xie, H. Yu, J. Lian, P. Wen, F. Zhang, H. Li, Y. Zeng, Z. Xiong, S. Liu, L. Zhou, Z. Huang, N. An, J. Wang, Q. Zheng, Y. Xiong, G. Wang, B. Wang, J. Wang, Y. Fan, R.R. da Fonseca, A. Alfaro-Núñez, M. Schubert, L. Orlando, T. Mourier, J.T. Howard, G. Huang, M.W. Bruford, X. Zhan, A. Dixon, M.F. Bertelsen, E. Derryberry, W. Warren, R.K. Wilson, S. Li, D.A. Ray, R.E. Green, S.J. O’Brien, D. Griffin, W.E. Johnson, D. Haussler, O.A. Ryder, E. Willerslev, G.R. Graves, P. Alström, J. Fjeldså, D.P. Mindell, S.V. Edwards, E.L. Braun, C. Rahbek, D.W. Burt, P. Houde, Y. Zhang, H. Yang, J. Wang, Avian Genome Consortium, E.D. Jarvis, M.T.P. Gilbert, J. Wang, Comparative genomics reveals insights into avian genome evolution and adaptation, Science. 346 (2014) 1311-1320.

[45] J. Xu, J.-T. Li, Y. Jiang, W. Peng, Z. Yao, B. Chen, L. Jiang, J. Feng, P. Ji, G. Liu, Z. Liu, R. Tai, C. 

Basis of Adaptive Evolution: The Survival of Amur Ide (Leuciscus waleckii) in an Extremely Alkaline Environment, Mol. Biol. Evol. 34 (2017) 145-159.

[46] M.R. Dorwart, N. Shcheynikov, D. Yang, S. Muallem, The solute carrier 26 family of proteins in epithelial ion transport, Physiology . 23 (2008) 104-114.

[47] G.D. Kavembe, P. Franchini, I. Irisarri, G. Machado-Schiaffino, A. Meyer, Genomics of adaptation to multiple concurrent stresses: insights from comparative transcriptomics of a Cichlid fish from one of earth's most extreme environments, the hypersaline soda Lake Magadi in Kenya, east Africa, J. Mol. Evol. 81 (2015) 90-109.

[48] D. Markovich, H. Murer, The SLC13 gene family of sodium sulphate/carboxylate cotransporters, Pflugers Arch. 447 (2004) 594-602.

[49] D. Basilio, K. Noack, A. Picollo, A. Accardi, Conformational changes required for $\mathrm{H}(+) / \mathrm{Cl}(-)$ exchange mediated by a CLC transporter, Nat. Struct. Mol. Biol. 21 (2014) 456-463. Regulating Lysosomal Function, Cell. 162 (2015) 1101-1112.

441 [51] M.P. Wilkie, C.M. Wood, The adaptations of fish to extremely alkaline environments, Comp. Biochem. Physiol. B Biochem. Mol. Biol. 113 (1996) 665-673. 
bioRxiv preprint doi: https://doi org/10 1101/825885; this version posted January 8,2020 . The copyright holder for this preprint (which was not certified by peer review) is the author/funder, who has granted bioRxiv a license to display the preprint in perpetuity. It is made available under aCC-BY-NC-ND 4.0 International license.

\section{Table}

446

447 Table 1. Summary of orthologous genes in five fish genomes and G. przewalskii transcriptomic 448 assemblies.

\begin{tabular}{llll}
\hline Species & Genes & Genes in orthologous groups & Unique orthologs \\
\hline Danio rerio & 52,089 & 29,232 & 17,001 \\
Gadus morhua & 22,100 & 16,884 & 16,390 \\
Takifugu rubripes & 47,841 & 25,137 & 16,071 \\
Oryzias latipes & 24,674 & 17857 & 15,877 \\
Oreochromis niloticus & 26,763 & 19,432 & 17,433 \\
Gymnocypris przewalskii & 137,539 & 74,107 & 16,379 \\
\hline
\end{tabular}


bioRxiv preprint doi: https://doi.org/10.1101/825885; this version posted January 8,2020 . The copyright holder for this preprint (which was not certified by peer review) is the author/funder, who has granted bioRxiv a license to display the preprint in perpetuity. It is made available under aCC-BY-NC-ND 4.0 International license.

451 Table 2. List of rapidly evolving ion transport genes in Gymnocypris przewalskii.

\begin{tabular}{lll}
\hline Gene name & Description & Adjusted P-value \\
\hline SCN3B & Sodium channel subunit beta-3 & 0.000076 \\
ATP1B2 & Sodium/potassium-transporting ATPase subunit beta-2 & 0.020563 \\
NALCN & Sodium leak channel non-selective protein & 0.029246 \\
SLC13A3 & Solute carrier family 13 member 3 & 0.002234 \\
TMEM175 & Transmembrane protein 175 & 0.002100 \\
CLCN7 & $\mathrm{H}(+) / C l(-)$ exchange transporter 7 & 0.000019 \\
TRPV6 & Transient receptor potential cation channel subfamily V member 6 & 0.022243 \\
MCU & Calcium uniporter protein, mitochondrial & 0.003946 \\
MICU2 & Calcium uptake protein 2, mitochondrial & 0.002823 \\
\hline
\end{tabular}




\section{$454 \quad$ Figure legends}

456 Figure 1. Genome-wide molecular evolutionary feature of six fish species. (A) Clipped species tree used 457 in this study. GPRZ, Gymnocypris przewalskii, DRER, Danio rerio, OLAT, Oryzias latipes, ONIL, 458 Oreochromis niloticus, TRUB, Takifugu rubripes, GMOR, Gadus morhua. (B) Barplot depicting the $459 \mathrm{dN} / \mathrm{dS}$ of concatenated supergenes in each fish species. (C) Violin plot depicting the dN/dS of each 460 coalescent orthologs in each species estimated by free-ratio model.

462 Figure 2. Expression feature of rapidly evolving ion transport genes (REITGs) in six tissues of $G$.

463 przewalskii. (A) Barplot depicting the expression level of nine REITGs in kidney, gill, liver, spleen, brain 464 and muscle tissues based on $\log _{10}(\mathrm{FPKM}+1)$ value which estimated from RNA-seq data. (B) Heatmap 465 depicting the expression level comparison of each REITG based on Log10(FPKM + 1) values. Tissue 466 type and gene name are shown on the $\mathrm{y}$-axis and $\mathrm{x}$-axis, respectively. Plot colors reflect the expression

467 level, ranging from low (blue) to high (red). (C) Barplot depicting the significantly enriched gene 468 ontology for differentially expressed REITGs. 


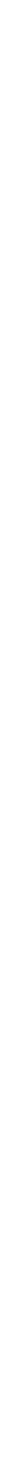


bioRxiv preprint doi: https://doi.org/10.1101/825885: this version posted Januarv 8. 2020. The copvriaht holder for this preprint (which was not certified/A peer review) is the author/funder: who has granted bioRxiv a license to dispilay the preprint in perpetuity. It is made available under

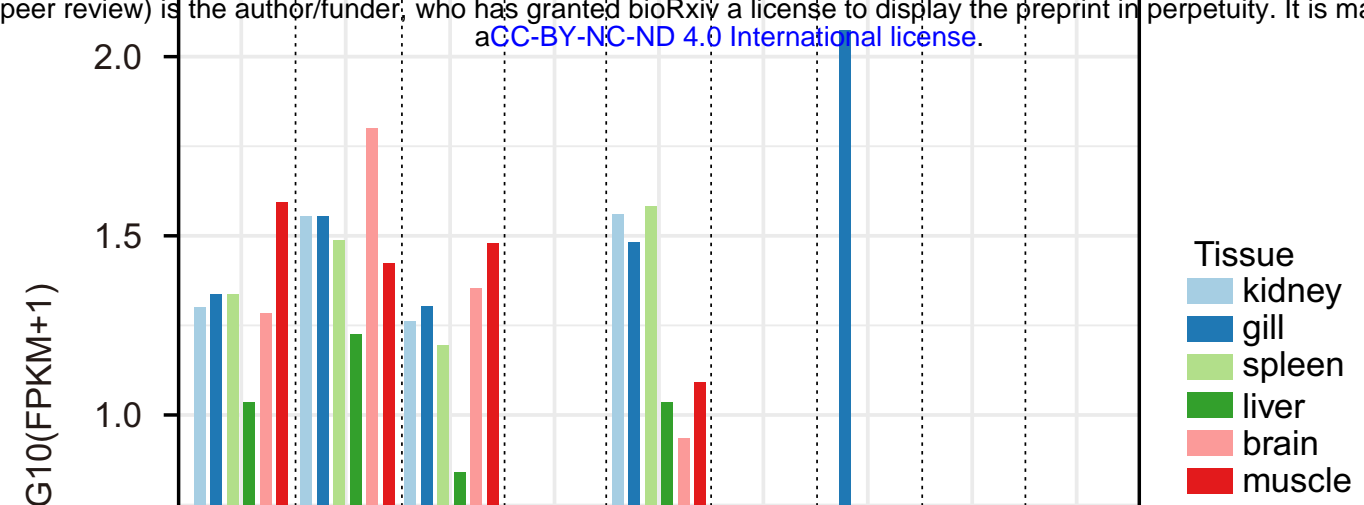

B

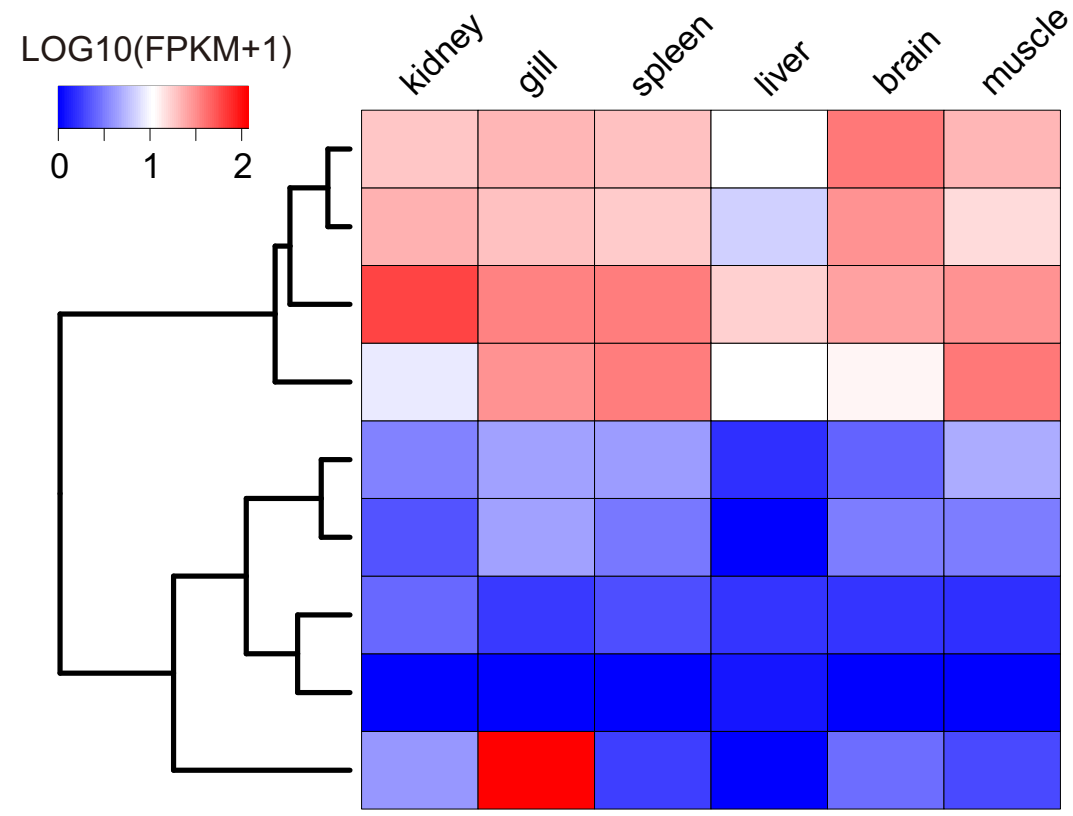

ATP1B2

MCU

CLCN7

NALCN

MICU2

SCN3B

TMEM175

TRPV6

SLC13A3

C

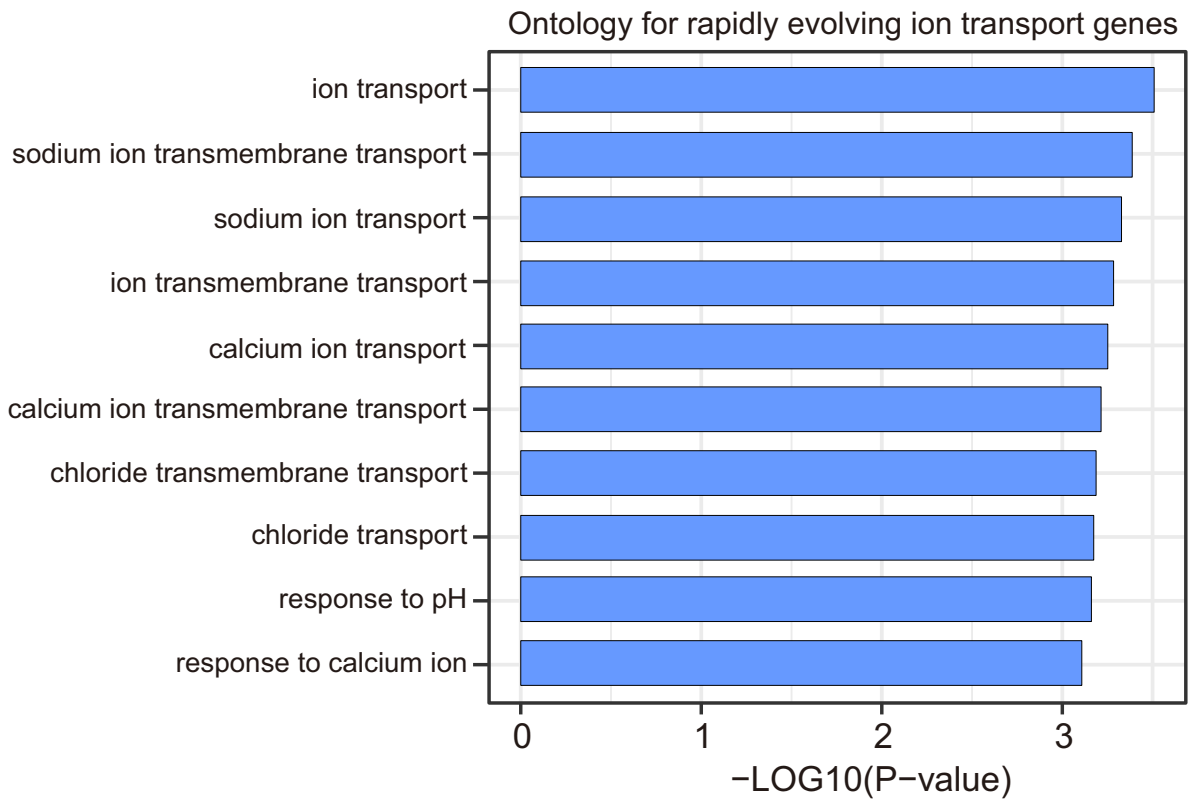

